\title{
REZENSIONEN
}

\section{Reformen und ihre Erfolgsbedingungen}

Wolf, Klaus Dieter (Hrsg.): Staat und Gesellschaft - fähig zur Reform? 23. wissenschaftlicher Kongress der Deutschen Vereinigung für Politische Wissenschaft, Nomos Verlagsgesellschaft, Baden-Baden 2007, 343 Seiten, €29,-.

Seit Beginn der neunziger Jahre ist der politische Diskurs in Deutschland durch anhaltendes Wehklagen über ausbleibende Reformen geprägt. Als die Gesellschaft für deutsche Sprache 1997 den Begriff „Reformstau“ zum Wort des Jahres ausrief, schienen sich die Kritiker bestätigt zu fühlen. Noch im selben Jahr sekundierte Bundespräsident Roman Herzog mit seiner Berliner „Ruck-Rede“ und wies auf die mangelnde Bereitschaft zu durchgreifenden Reformen in weiten Teilen von Politik und Gesellschaft hin.

Am Wegesrand politischer Reformen warten seit jeher die wohlmeinenden Diktatoren mit Vorschlägen für intelligentere Lösungen und die Stenographen des Scheiterns, um fehlgeschlagene Reformen für die Nachwelt zu dokumentieren. Nicht selten offenbart das Lamento über nicht stattfindende Reformen ein tiefgreifendes Missverständnis über die Funktionsbedingungen des politisch-administrativen Systems der Bundesrepublik. Durch die Diffusion politischer Gestaltungsmacht entstehen in Deutschland starke Verhandlungsund Kooperationszwänge zwischen den beteiligten Akteuren, um adäquate Problemlösungen herbeizuführen. Konstitutionell verankerte Veto-Positionen, kollektiv organisierte Interessen sowie verschachtelte Entscheidungswege reduzieren nicht nur die Möglichkeiten hierarchischer Steuerung der Politik, sondern lassen insgesamt nur begrenzte Veränderungen zu. Zwar führen diese Randbedingungen politischen Handelns zu einer stark eingeschränkten Binnensouveränität des Staates, doch ermöglicht die komplexe Architektur des politischen Systems sehr wohl Reformaktivitäten. ${ }^{1}$

Auf ihrem 23. wissenschaftlichen Kongress hat sich die Deutsche Vereinigung für Politische Wissenschaft im Herbst 2006 mit den Möglichkeiten, Grenzen und Maßstäben politischer Reformen beschäftigt, dessen Ergebnisse der vorliegende Band versammelt. Dabei wird der Analysegegenstand aus drei unterschiedlichen Perspektiven beleuchtet. Unter der Überschrift „Reform des Staates“ wird der Wandel von Staatlichkeit thematisiert und gefragt, unter welchen Bedingungen sich die Institutionen des Staates verändern lassen. Mit Blick auf spezifische Politikfelder widmet sich der Abschnitt „Reform im Staat“ politischen Veränderungen auf der policy-Ebene. Das dritte Kapitel „Reform ohne Staat“ geht der Frage nach, wie sich Politik unter den Bedingungen grenzüberschreitender quasi-staatlicher Arrangements und damit jenseits des Nationalstaates verändern kann. Im letzten Abschnitt wird erörtert, welchen Beitrag die Politikwissenschaft für erfolgreiche Reformpolitik leisten kann.

1 Vgl. Peter J. Katzenstein, Policy and politics in West Germany. The growth of a semi-sovereign state, Philadelphia 1987. Daneben auch Simon Green / William E. Paterson (Hrsg.), Governance in contemporary Germany. The semi-sovereign state revisited, Cambridge 2005. 
Die politische Diskussion in der Bundesrepublik ist von der Annahme geprägt, dass umfassende, strukturverändernde Politik kaum möglich sei. Stimmt man dieser Auffassung zu, dann wäre - wie Klaus Dieter Wolf zu Beginn richtig feststellt - „das eigentlich Erklärungsbedürftige gar nicht die Reformunfähigkeit, sondern die Reformen, die gleichwohl stattfinden" (S. 19). In diesem Sinne beleuchten Philipp Genschel, Stephan Leibfried und Bernhard Zangl unter der Überschrift „Reform des Staates“ die Wandlungsprozesse des souveränen und nach innen hierarchisch integrierten Nationalstaates. Durch Prozesse der Internationalisierung und Privatisierung - begleitet von gewandelten normativen Leitbildern - entstehen Formen neuer Staatlichkeit. Dabei betreten zunehmend private, quasi-staatliche und substaatliche Akteure die politische Arena und wirken an der Erbringung von Dienstleistungen und öffentlichen Gütern mit. Ausgehend von der Diagnose einer „Zerfaserung von Staatlichkeit“ (S. 40) und neuen Akteurskonstellationen steht die Kardinalfrage im Mittelpunkt, wer sich „reformfreudig“ (S. 43) zeigen muss. Es wäre zu kurzsichtig, lediglich Politikversagen und Steuerungsschwächen zu ermitteln; zunächst kommt es darauf an, Zuständigkeiten zu benennen, um auf diese Weise die entsprechenden Verantwortlichkeiten für nicht erfolgte oder misslungene Reformen zuzuweisen. Insgesamt kommt es darauf an, die vollständigen „Strukturen moderner Politik“ (S. 44) zu berücksichtigen und Abschied von der Vorstellung zu nehmen, der Staat könne als dominante Steuerungsinstanz oder gar als autoritärer Zuchtmeister in Erscheinung treten.

Im Abschnitt „Reform im Staat“ untersucht Katja Patzwaldt, welchen Beitrag Politikberatung zu den Reformen am Arbeitsmarkt der rot-grünen Bundesregierung leisten konnte. Unter Berücksichtigung der Benchmarkinggruppe des Bündnisses für Arbeit und der Hartz-Kommission fragt sie, inwieweit Expertenberatung hilfreich war, um Mehrheiten für Innovationen auf dem Feld der Arbeitsmarktpolitik zu organisieren. Gestützt auf eine Reihe von Interviews mit beteiligten Akteuren aus Politik und Wissenschaft resümiert die Autorin, dass die in der Benchmarkinggruppe erarbeiteten wissenschaftlichen Begleitkonzepte „der Partei Unausweichlichkeiten des Regierens und der Globalisierung verdeutlichen und zugleich Inhalte für ihre Erneuerung auf dem ,Dritten Weg' bieten [sollten]“ (S. 218). Mit Blick auf die Hartz-Kommission stellt Patzwaldt fest, dass es ihr gelang, neue Wege der Legitimierung und eine breitere Verankerung von weitreichenden Reformprogrammen in der Gesellschaft zu finden. Insgesamt bleibt festzuhalten, dass die rot-grüne Regierung neuartige Formen der wissenschaftsgestützten Politikberatung zu etablieren versucht hat. Zwar konnte der politische Diskurs durch externe Beratung an Objektivität und Rationalität hinzugewinnen, doch gab es oftmals Widerstand bei den Akteuren, die ungünstige Machtverschiebungen im Prozess der Politikformulierung befürchteten.

Im letzten Abschnitt des Bandes - überschrieben mit „Reform (in) der Politikwissenschaft“ - beleuchtet Michael Th. Greven, was eine moderne, aufgeklärte Policy-Forschung zu einem besseren Verständnis von Reformbedingungen in der Politik beitragen kann. Während in früheren Jahren eine „technokratisch-gouvernementale Problemlösungsperspektive" (S. 333) vorherrschte, geht es heute nicht mehr bloß um die Politik auf der einen und einen amorphen Adressatenkreis auf der anderen Seite. Die Politikfeldanalyse buchstabiert das politische Geschehen vielmehr als zyklischen Ablauf von Problemwahrnehmung, Programmformulierung und Implementation. Erst durch die Berücksichtigung von Adressaten, die Anhörung von Betroffenen, die Mobilisierung externen Sachverstandes sowie durch eine breite gesellschaftliche Verankerung von Reformen wird das Prozesshafte von Politik deutlich. Auch wenn Entscheidungen durch beteiligungsorientierte Verfahren er- 
gänzt werden, so resümiert Greven, „dominiert am Ende doch die legitimierte Amtsgewalt oder Mehrheitsentscheidung“" (S. 338).

Insgesamt handelt es sich bei dem vorliegenden Tagungsband um eine interessante und lesenswerte Zusammenstellung von Beiträgen, die sich auf unterschiedliche Weise dem Phänomen politische Reformen nähern. Im Kontext der Reformdiskussion liegt dem Titel des Buches - Staat und Gesellschaft - implizit eine gängige Unterscheidung aus dem Bereich der politischen Steuerungswissenschaft zugrunde: Von Bedeutung ist damit nicht nur die Steuerungsfähigkeit (Staat), sondern gleichzeitig die Steuerbarkeit unterschiedlicher Teilsektoren (Gesellschaft), um Veränderungen wirkungsvoll ins Werk setzen zu können. Trotz eines positiv ausfallenden Gesamturteils verbleiben einige einschränkende Anmerkungen. So hätte es das Bild abgerundet, wenn die Rolle der Parteien und des Bundestages als Promotoren von Innovationen und Reformen stärker beleuchtet worden wäre. Bei der Auswahl der einzelnen Politikfelder hätte man sich gewünscht, dass insbesondere jene Bereiche thematisiert worden wären, die seit vielen Jahren kontrovers diskutiert werden und in der Öffentlichkeit symptomatisch für den deutschen „Reformstau“ stehen (vor allem das Steuersystem und der Gesundheitssektor). Gerade im Abschnitt Reform ohne Staat hätte man gern erfahren, ob erfolgreiche Veränderungen in Politik und Gesellschaft nicht auch im Gewand nicht-intendierter Nebenfolgen staatlichen Handelns daherkommen können. Am Ende sei das Buch insbesondere all jenen Beobachtern und Kommentatoren als aufschlussreiche Lektüre empfohlen, die zwar gescheiterte Reformen zur Kenntnis nehmen, die systemeigenen Erfolgsbedingungen politischer Reformanstrengungen in der Bundesrepublik jedoch kaum berücksichtigen.

Helge F. Jani

\section{Edierte Protokolle der Bundestagsfraktion der Grünen}

\section{Boyer, Josef und Helge Heidemeyer (Hrsg.): Die Grünen im Bundestag. Sitzungsprotokolle} 1983-1987, Droste Verlag, Düsseldorf 2008, 2 Halbbände, 1137 Seiten, € 140,-.

Die Grünen sind historisch geworden. Vorbei sind die Zeiten, da man ausrechnen musste, mit welcher Wahrscheinlichkeit die Partei über die Fünf-Prozent-Hürde kommen werde. Von den frühesten Grünen Listen an gerechnet, sind die Grünen nun schon mehr als vierzig Jahre präsent, gerade haben sie ihr 25jähriges Jubiläum als Bundestagsfraktion gefeiert. Im Fünfparteiensystem der Bundesrepublik erwachsen ihnen neue Chancen. Sie sind als eine der Grundströmungen des Parteiensystems nicht mehr wegzudenken.

Immer noch klagt das Feuilleton, sie hätten sich zu sehr „angepasst“. Jetzt gibt es Gelegenheit, die wilde Geschichte der Grünen noch einmal in ihren Anfängen nachzulesen. Die Kommission für Geschichte des Parlamentarismus und der politischen Parteien hat verdienstvollerweise die Sitzungsprotokolle der grünen Bundestagsfraktion 1983 bis 1987 herausgebracht, ergänzt um eine Fülle von Dokumenten, die Licht auf das Innere grüner Prozesse werfen. Man sollte die Chance nutzen, um zu verstehen, dass es für die Grünen gut war, nicht bei ihren Kinderkrankheiten stehen zu bleiben. 